\title{
Genistein induces degradation of mutant huntingtin in fibroblasts from Huntington's disease patients
}

\author{
Karolina Pierzynowska ${ }^{1} \cdot$ Lidia Gaffke $^{1} \cdot$ Zuzanna Cyske $^{1} \cdot$ Grzegorz Węgrzyn $^{1}$ (D)
}

Received: 17 February 2019 / Accepted: 1 March 2019 / Published online: 9 March 2019

(C) The Author(s) 2019

\begin{abstract}
Mutations in the HTT gene, consisting of expansion of CAG triplets, cause the Huntington's disease (HD), one of the major neurodegenerative disorders. Formation of aggregates of mutant huntingtin (mHTT, the product of the mutant HTT gene) leads to cellular dysfunctions, and subsequent neurodegeneration which manifest clinically as motor abnormalities and cognitive deficits. We recently used immortalized HEK-293 cells expressing the 1st exon of the mutant HTT gene as a cellular model of HD, and showed that the stimulation of autophagy by genistein corrected the mutant phenotype. However, effects of genistein on HD patient-derived cells remained unknown. In this report, we demonstrated that genistein also instigated degradation of mHTT in fibroblasts derived from HD patients. This was assessed as a significant decrease in the levels of HTT in HD fibroblasts measured by Western-blotting, and the disappearance of intracellular mHTT aggregates in cells observed by fluorescent microscopy. Fibroblasts derived from control persons were not affected by genistein treatment. These results indicate that genistein can improve HD phenotype in patient-derived cells, and substantiates the need for further studies of this isoflavone as a potential therapeutic agent.
\end{abstract}

Keywords Huntington's disease $\cdot$ Genistein $\cdot$ Huntingtin $\cdot$ Protein degradation $\cdot$ Protein aggregates $\cdot$ Autophagy

\section{Introduction}

Huntington's disease (HD) is an autosomal dominant genetic disorder which is manifested clinically by specific symptoms including chorea, psychiatric disturbances and cognitive decline (Morreale 2015). This neurodegenerative disease is fatal, as no effective treatments are currently available (Shannon and Fraint 2015).

HD is caused by mutations in the HTT gene, coding for huntingtin (HTT), which result in the expansion of CAG repeats (Morreale 2015). This expansion results in the formation of a long tract of glutamine residues (the polyQ tract) in the mutant huntingtin protein (mHTT). Due to misfolding of polyQ-containing domains, $\mathrm{mHTT}$ forms aggregates that cause cellular dysfunctions resultant in progressive neurodegeneration (Morreale 2015). It is generally accepted that 40 or more CAG repeats in the 1st exon of HTT lead to full penetrance of

Grzegorz Węgrzyn

grzegorz.wegrzyn@biol.ug.edu.pl

1 Department of Molecular Biology, University of Gdańsk, Wita Stwosza 59, 80-308 Gdańsk, Poland the mutation and the occurrence of HD-specific symptoms (as stated by American College of Medical Genetics and the American Society of Human Genetics).

Although no effective treatments of HD are available, various approaches to improve cell viability by inhibiting expression of the mutant HTT allele, the inactivation of mHTT, and/or the degradation of mHTT were tested (Shannon and Fraint 2015). It has been suggested recently that stimulation of the autophagy process might be the most promising strategy for development of effective therapies for diseases caused by accumulation of pathological macromolecules, including mHTT (Guo et al. 2018; Pierzynowska et al. 2018a). However, the vast majority of autophagy stimulators characterized to date do not meet the criteria to be used as long-term therapeutics crucial for the treatment of neurodegenerative diseases (Yang et al. 2013). Particularly, dose- and/or duration-dependent overactivation of autophagy might potentially cause severe adverse effects. Genistein (trihydroxyisoflavone or 5, 7-dihydroxy-3- (4hydroxyphenyl)-4H-1-benzopyran-4-one), a potent autophagy stimulator, might be a putative drug for the treatment of neurodegenerative diseases, because it crosses the blood-brain-barrier, and is safe when administered even at high doses (like $150 \mathrm{mg} / \mathrm{kg} /$ day) for several months to animals and humans 
(Malinowska et al. 2009, 2010; Kim et al. 2013; Moskot et al. 2014). Our recent studies, using immortalized HEK-293 cells transiently transfected with a plasmid expressing the 1st exon of the $m H T T$ gene as a cellular model of HD showed that genistein-mediated induction of autophagy corrected the HD phenotype (Pierzynowska et al. 2018b). Although these results are promising, a demonstration of beneficial effects of genistein in HD patient's cells is necessary.

Although HD primarily affects the brain, $H T T$ is ubiquitously expressed also in peripheral cells (Sharp et al. 1995; Kegel et al. 2002). Moreover, HD fibroblasts manifest cellular dysfunctions similar to neural HD cells (Sassone et al. 2009; Marchina et al. 2014; Petersen et al. 2014; Jedrak et al. 2017a). Since fibroblasts can be readily obtained from patients, and they can be easily cultured under laboratory conditions, we decided to assess the efficacy of genistein in HD patient-derived fibroblasts.

\section{Materials and methods}

\section{Formal and ethical issues}

This study was conducted according to the tenets of the Helsinki Declaration, and it was approved by the local Ethics Committee of the Medical University of Gdansk (NKEBN/254/2011 and NKEBN/254-431/2012). Written informed consents were obtained from all donors of the biological material, both HD patients and persons from the control group, prior to the study procedures.

\section{Human subjects}

Biological material from four HD patients and four age- and sex-matched controls was used in this study (information about the written informed consents is provided in the previous subsection). The Unified Huntington's Disease Rating Scale (UHDRS) was used to assess HD severity (Huntington Study Group 1996). Psychiatric features were assessed by the Clinical Global Impressions scale (CGI), and the functional assessment was measured by the Total Functional Capacity scale (TFC), as described previously (Jeedrak et al. 2018). Characteristics of the HD patient and control groups is presented in Table 1.

\section{Cell cultures}

Biopsies were taken from the forearm skin, and fibroblast lines were established as described previously (Jedrak et al. 2017a, 2017b, 2018). Fibroblasts were cultured on 10-cm plates in DMEM (Thermo Fisher Scientific Inc., Paisley, UK) supplemented with 10\% FBS (Thermo Fisher Scientific Inc., Paisley, UK) and 1\% antibiotic/antimycotic solution (Sigma-Aldrich Co. LLC., St. Louis, USA) at $37{ }^{\circ} \mathrm{C}$ under a humidified atmosphere of $95 \%$ air $/ 5 \% \mathrm{CO}_{2}$.
Table 1 Characteristics of patients and control subjects at the time of skin biopsy

\begin{tabular}{|c|c|c|c|c|c|c|}
\hline \multirow[t]{2}{*}{ Subject } & \multirow[t]{2}{*}{ Sex } & \multirow{2}{*}{$\begin{array}{l}\text { Age } \\
\text { (y) }\end{array}$} & \multirow{2}{*}{$\begin{array}{l}\text { No. of CAG } \\
\text { repeats }\end{array}$} & \multicolumn{2}{|c|}{ Rating scale } & \multirow{2}{*}{$\begin{array}{l}\text { Duration of } \\
\text { motor symptoms (y) }\end{array}$} \\
\hline & & & & CGI & TFC & \\
\hline Control 1 & M & 51 & NT & NA & NA & NA \\
\hline Control 2 & M & 50 & NT & NA & NA & NA \\
\hline Control 3 & M & 51 & NT & NA & NA & NA \\
\hline Control 4 & M & 43 & NT & NA & NA & NA \\
\hline HD 1 & M & 41 & 43 & 3 & II & 17 \\
\hline HD 2 & $\mathrm{~F}$ & 45 & 43 & 4 & II & 4 \\
\hline HD 3 & M & 54 & 43 & 5 & III & 13 \\
\hline HD 4 & M & 49 & 42 & 4 & I & 2 \\
\hline
\end{tabular}

Abbreviations: CGI, Clinical Global Impressions scale; TFC, Total Functional Capacity scale; M, male; F, female; NT, not tested; NA, not applicable

\section{Reagents}

Genistein (99\% purity; \#446-72-0) was purchased from Pharmaceutical Research Institute, Warsaw, Poland). It was dissolved in DMSO at stock concentrations of 30,60, and $100 \mathrm{mM}$, and stored at $-20^{\circ} \mathrm{C}$. The following antibodies were used: monoclonal mouse anti-human huntingtin clone mEM48 (\#MAB5374 for immunofluorescence), and clone 1HU-4C8 (\#MAB2166 for Western-blotting) (SigmaAldrich, city, Munich, Germany); goat anti-mouse conjugated with Alexa Fluor 488 (\#A-11001, Thermo Fisher Scientific, city, Waltham, Massachusetts, USA); anti- $\beta$-actin conjugated with horse radish peroxidase (\#A3854, Sigma Aldrich, city, Munich, Germany).

\section{Immunoblotting}

$6 \times 10^{5}$ cells were passaged on plates $(10 \mathrm{~cm}$ in diameter), and allowed to attach overnight. Cells were treated with either DMSO (final concentration of $0.1 \%$; control cells) or 30 , 60 , and $100 \mu \mathrm{M}$ genistein for $48 \mathrm{~h}$. Cells were lysed with a solution containing $1 \%$ Triton X-100, $0.5 \mathrm{mM}$ EDTA, $150 \mathrm{mM} \mathrm{NaCl}, 50 \mathrm{mM}$ Tris, $\mathrm{pH} 7.5$, and a mixture of protease and phosphatase inhibitors (Roche Applied Science, Penzberg, Germany; \#05892791001 and \#11873580001), and cleared by centrifugation. Proteins were separated using the WES system (WES Automated Western Blots with Simple Western; ProteinSimple, San Jose, California, USA), with 12 $230 \mathrm{kDa}$ Separation Module (\#SM-W003), and detected with Anti-Mouse Detection Module (\#DM-002), according to the manufacturer's instruction. Staining with anti- $\beta$-actin antibody (1:25000) was used as an internal control to normalize the amounts of huntingtin. 


\section{Fluorescence microscopy}

$4 \times 10^{4}$ fibroblasts were passaged on coverslips in 12-well plates, and allowed to attach overnight. Cells were treated with either DMSO (control cells), or 30,60 , and $100 \mu \mathrm{M}$ genistein for $48 \mathrm{~h}$. The cells were fixed with $2 \%$ paraformaldehyde in phosphate buffered saline (PBS), and rinsed with $0.1 \%$ Triton X-100 in PBS. Then, the samples were blocked with 5\% BSA and 1.5\% glycine in PBS for $1 \mathrm{~h}$. Following overnight incubation with primary antibody $(1: 1000)$ dissolved in PSB, the cells were rinsed with PBS 3 times, and incubated with secondary antibody dissolved in PBS (1:4000) for $2 \mathrm{~h}$. After washing with PBS (5 times) coverslips were affixed to glass slides with a mounting medium, and next day, they were analyzed using the Nikon Eclipse E800 microscope.

\section{Statistical analysis}

Data are presented as means \pm SDs. Comparisons between two groups were performed by the Student's $t$ test, and comparison between several groups were tested by ANOVA. Statistical differences were considered significant at $p<0.05$.

\section{Approval and accordance}

All experimental protocols were approved by the Head of Department of Molecular Biology and Dean of Faculty of Biology, according to the procedures described in guidelines and regulations of the Vice-rector for Research of University of Gdańsk.
Fig 1 Genistein reduces the number of mHTT aggregates in human fibroblasts. Cultures of fibroblasts derived from four patients suffering from Huntington's disease (HD) and four healthy persons (WT) were treated with either DMSO (control) or 30, 60 and $100 \mu \mathrm{M}$ genistein, for $48 \mathrm{~h}$. Cells were immuno-stained with antihuntingtin antibody, secondary antibody conjugated with Alexa Fluor 488, and analyzed by fluorescent microscopy at $1000 \mathrm{x}$ magnification. The number of aggregates per $100 \mu \mathrm{m}^{2}$ were counted in 100 cells per culture. The experiments were performed in triplicates. Panel A demonstrates representative cells from each group. Panel B shows quantitative analysis of the number of aggregates, where bars represent mean values $\pm \mathrm{SD}$, and asterisks indicate statistically significant differences $(p<0.05)$ between WT and HD groups. Panel C demonstrates association of mHTT with cytoskeletal fibers in fibroblasts derived from HD patient. In panels $\mathrm{A}$ and $\mathrm{C}$, white scale bars indicate $10 \mu \mathrm{m}$ a

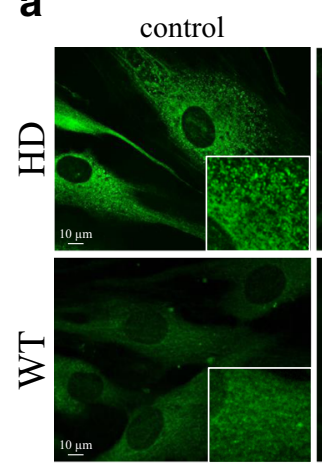

$30 \mu \mathrm{M}$ genistein

$60 \mu \mathrm{M}$ genistein

$100 \mu \mathrm{M}$ genistein

b
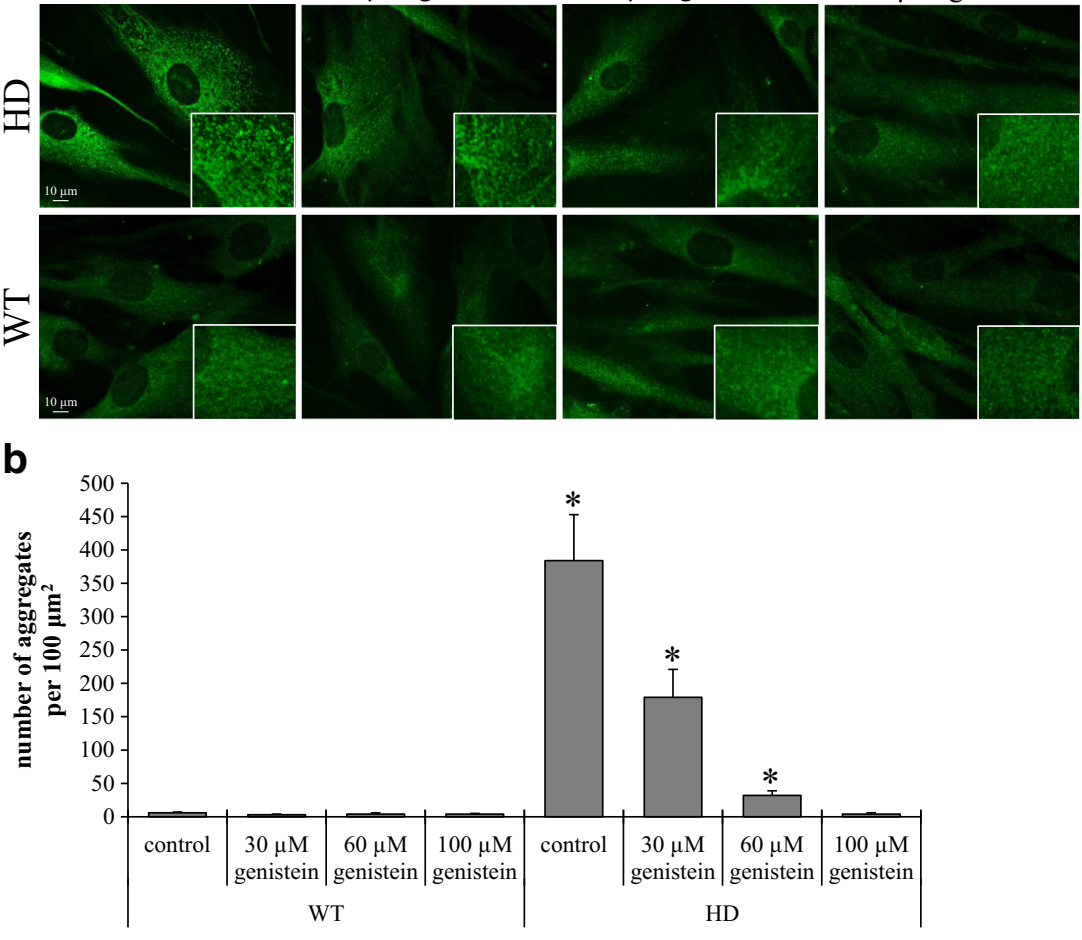

C
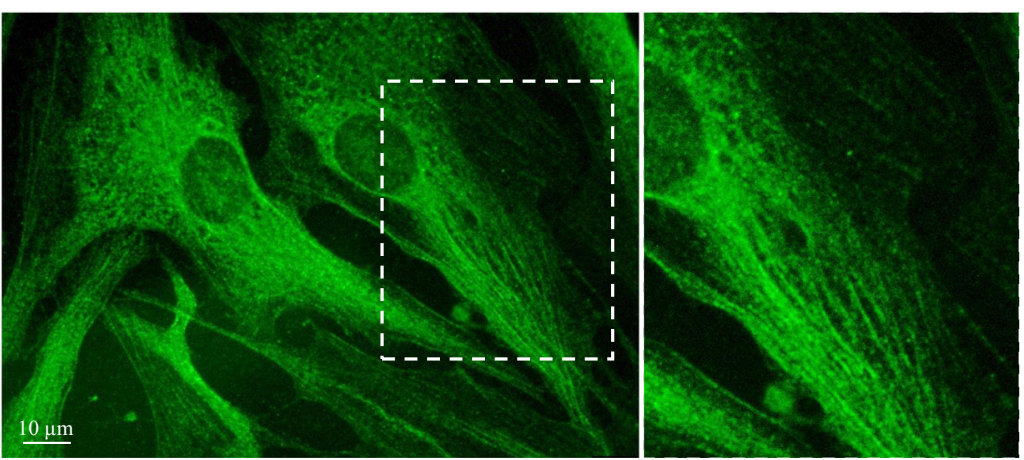


\section{Results}

To investigate effects of genistein on patient-derived HD cells, we tested fibroblast cell lines obtained from four patients with confirmed HD diagnosis, and four volunteers without any HD symptoms and with no HD familial history as the control group. The experiments were performed with all cell lines, and each experiment was repeated at least 3 times. Then, results of each group (each set of 4 cell lines) were combined and analyzed.

In our previous studies (Pierzynowska et al. 2018b), we used HEK-293 cells transiently transfected with a plasmid encoding a fusion protein consisting of the 1st exon of HTT bearing 74 CAG repeats and the GFP protein. Therefore, when using anti-GFP antibodies, it was possible to specifically monitor levels of mHTT, without signals from endogenous HTT encoded by chromosomal HTT. In contrast, in patientderived fibroblasts, both forms, i.e., HTT and mHTT, are present, because HD patients are heterozygotic for the HTT gene. The anti-huntingtin antibody detects both HTT and mHTT as the proteins differ only in the length of glutamine repeats. Thus, the major difference between control and HD cells should be reflected by the level of total HTT (HTT + mHTT), and the number of intracellular aggregates.

As expected, when control fibroblasts were studied using fluorescent microscopy, HTT was uniformly distributed, and the fluorescent signals were moderate (Fig. 1). In contrast, HD fibroblasts revealed increased intensity of HTT staining that formed multiple aggregates apparently composed of mHTT. These aggregates tended to associate with the cytoskeletal fibers (Fig. 1).
The addition of genistein at different concentrations had no significant effect on control cells. However, in HD fibroblasts, HTT-specific signals were reduced significantly in a dosedependent manner (Fig. 1). At the highest genistein concentration, the intensity of HTT signals approached the intensity seen in control fibroblasts.

Western-blot analysis revealed significantly higher levels of HTT in untreated HD relative to control cells (Fig. 2). The exposure of HD fibroblasts to genistein decreased cellular HTT levels, in the dose-dependent manner. At 60 and $100 \mu \mathrm{M}$ genistein, HTT levels dropped to the levels in control fibroblasts.

\section{Discussion}

The pathology of many neurodegenerative diseases feature the formation of protein aggregates (Aguzzi and O'Connor 2010). Therefore, one might suggest that enhanced degradation of protein aggregates would be the most effective way to treat these diseases. In fact, we (Pierzynowska et al. 2018a) and others (Guo et al. 2018) have proposed that autophagy, a major processes of protein degradation in cells, could be a promising target for therapeutic intervention to combat neurodegeneration.

HD can be considered a model for this group of neurodegenerative disorders (Morreale 2015). The expansion of CAG repeats in the HTT gene results in the generation of the pathogenic protein, mHTT, which accumulates and forms insoluble aggregates. Our recent studies showed that genistein, an efficient and safe stimulant of autophagy can correct HD phenotype in HEK-293 cells transfected with the HTT gene
Fig 2 Genistein normalizes levels of HTT in HD fibroblasts. Cultures of fibroblasts derived from four HD patients (HD) and four healthy persons (WT) were treated with either DMSO (control) or 30, 60 and $100 \mu \mathrm{M}$ genistein, for $48 \mathrm{~h}$. Cellular proteins were separated and detected using the WES system. Four HD and four WT cell lines were used, and the analyses were performed in triplicates. Representative blots are shown in the upper panel, and quantitative analysis is presented in the lower panel. Bars represent mean values \pm SD from three independent experiments. Asterisks indicate statistically significant differences $(p<0.05)$ between WT control and HD groups
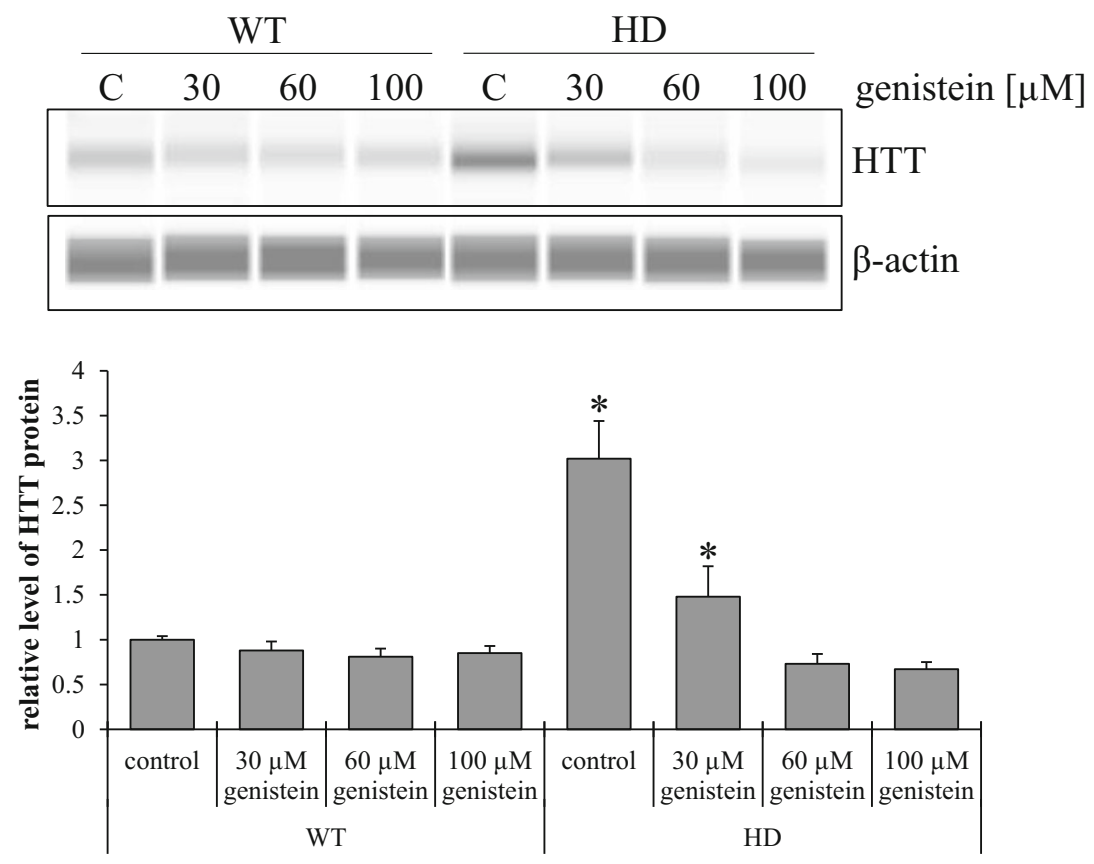
containing 74 CAG repeats (Pierzynowska et al. 2018b). This experimental system allowed for high level production of the mutant polypeptide and the formation of large amounts of intracellular aggregates, and thus, it was useful in testing efficiency of genistein action and in investigating the underlying mechanisms. However, the question remained whether genistein can effectively correct the HD phenotype of patientderived cells. Therefore, we tested fibroblast lines derived from HD patients and controls. This model was chosen because HD fibroblast are unmodified cells derived directly from patients, and because they possess common features with HD neural cells (Sassone et al. 2009; Marchina et al. 2014; Petersen et al. 2014; Jędrak et al. 2017a).

Our present data clearly indicate that genistein is effective in normalizing HTT levels in HD fibroblasts as demonstrated by immunofluorescence and Westernblotting experiments. Because HTT levels were unchanged after treatment of control fibroblasts, we conclude that genistein induced specific degradation of mHTT, with no significant influence on normal HTT. This is in concordance with our previous studies in HEK-293 cells showing genistein-induced degradation of mHTT with $74 \mathrm{Q}$ residues, but no degradation of mHTT with 23 Q residues (Pierzynowska et al. 2018b).

We observed that in HD fibroblasts, mHTT formed aggregates that associated with cytoskeletal fibers. Such results are compatible with recently reported observations that HTT can be associated with isoforms of $\alpha$-actinin that bind actin filaments, and that mHTT localizes specifically to actin stress fibers (Tousley et al. 2019). Again, treatment with genistein resulted in disappearance of aggregates, and HTT distribution resembling the distribution in healthy cells.

In summary, our results demonstrate that genistein can correct HD phenotype in patient-derived cells. Of note, genistein might also be effective in other neurodegenerative diseases, e.g., Alzheimer's disease (Pierzynowska et al. 2019), and Parkinson's disease (Wu et al. 2018). Therefore, it is tempting to suggest that genistein, acting as an autophagy stimulator, can be considered a potential therapeutic agent for various neurodegenerative diseases that feature accumulation of misfolded proteins. Altogether, these results provide a rationale for further studies aimed at developing genistein-based therapy for HD and possibly other neurodegenerative disorders.

Acknowledgements We thank Prof. Jarosław Sławek for performing skin biopsies and for providing clinical data. This work was supported by National Science Center, Poland (project grant no. 2017/25/N/NZ2/ 00812), and University of Gdansk (project grant no. 538-L140-B137-18).

Author contributions K.P. participated in planning the study, data analysis, and drafting the manuscript. K.P. and L.G. performed all experiments. Z.C. assisted and helped in all experiments. G.W. supervised the study, participated in data analysis, and drafted the manuscript.

\section{Compliance with ethical standards}

Conflict of interest The use of genistein in treatment of Huntington's disease is a subject of patent application (no. P.417983, submitted to Polish Patent Office). No other conflicts of interest are declared by the authors.

Open Access This article is distributed under the terms of the Creative Commons Attribution 4.0 International License (http:// creativecommons.org/licenses/by/4.0/), which permits unrestricted use, distribution, and reproduction in any medium, provided you give appropriate credit to the original author(s) and the source, provide a link to the Creative Commons license, and indicate if changes were made.

Publisher's note Springer Nature remains neutral with regard to jurisdictional claims in published maps and institutional affiliations.

\section{References}

Aguzzi A, O’Connor T (2010) Protein aggregation diseases: pathogenicity and therapeutic perspectives. Nat Rev Drug Discov 9:237-248

Guo F, Liu X, Cai H, Le W (2018) Autophagy in neurodegenerative diseases: pathogenesis and therapy. Brain Pathol 28:3-13

Huntington Study Group (1996) Unified Huntington's disease rating scale: reliability and consistency. Mov Disord 11:136-142

Jędrak P, Krygier M, Tońska K et al (2017a) Mitochondrial DNA levels in Huntington disease leukocytes and dermal fibroblasts. Metab Brain Dis $32: 1237-1247$

Jędrak P, Sowa N, Barańska S, Węgrzyn G (2017b) Characterization of conditions and determination of practical tips for mtDNA level estimation in various human cells. Acta Biochim Pol 64:699-704

Jędrak P, Mozolewski P, Węgrzyn G, Więckowski MR (2018) Mitochondrial alterations accompanied by oxidative stress conditions in skin fibroblasts of Huntington's disease patients. Metab Brain Dis 33:2005-2017

Kegel KB, Meloni AR, Yi Y et al (2002) Huntingtin is present in the nucleus, interacts with the transcriptional corepressor $\mathrm{C}$-terminal binding protein, and represses transcription. J Biol Chem 277: 7466-7476

Kim KH, Dodsworth C, Paras A, Burton BK (2013) High dose genistein aglycone therapy is safe in patients with mucopolysaccharidoses involving the central nervous system. Mol Genet Metab 109:382385

Malinowska M, Wilkinson FL, Bennett W, Langford-Smith KJ, O'Leary HA, Jakobkiewicz-Banecka J, Wynn R, Wraith JE, Wegrzyn G, Bigger BW (2009) Genistein reduces lysosomal storage in peripheral tissues of mucopolysaccharide IIIB mice. Mol Genet Metab 98: 235-242

Malinowska M, Wilkinson FL, Langford-Smith KJ, Langford-Smith A, Brown JR, Crawford BE, Vanier MT, Grynkiewicz G, Wynn RF, Wraith JE, Wegrzyn G, Bigger BW (2010) Genistein improves neuropathology and corrects behaviour in a mouse model of neurodegenerative metabolic disease. PLoS One 5:e14192

Marchina E, Misasi S, Bozzato A et al (2014) Gene expression profile in fibroblasts of Huntington's disease patients and controls. J Neurol Sci 337:42-46

Morreale MK (2015) Huntington's disease: looking beyond the movement disorder. Adv Psychosom Med 34:135-142

Moskot M, Montefusco S, Jakóbkiewicz-Banecka J, Mozolewski P, Węgrzyn A, Di Bernardo D, Wegrzyn G, Medina DL, Ballabio A, Gabig-Cimińska M (2014) The phytoestrogen genistein modulates 
lysosomal metabolism and transcription factor EB (TFEB) activation. J Biol Chem 289:17054-17069

Petersen MH, Budtz-Jørgensen E, Sørensen SA et al (2014) Reduction in mitochondrial DNA copy number in peripheral leukocytes after onset of Huntington's disease. Mitochondrion 17:14-21

Pierzynowska K, Gaffke L, Cyske Z, Puchalski M, Rintz E, Bartkowski M, Osiadły M, Pierzynowski M, Mantej J, Piotrowska E, Węgrzyn G (2018a) Autophagy stimulation as a promising approach in treatment of neurodegenerative diseases. Metab Brain Dis 33:989-1008

Pierzynowska K, Gaffke L, Hać A, Mantej J, Niedziałek N, Brokowska J, Węgrzyn G (2018b) Correction of Huntington's disease phenotype by genistein-induced autophagy in the cellular model. NeuroMolecular Med 20:112-123

Pierzynowska K, Podlacha M, Gaffke L, Majkutewicz I, Mantej J, Wegrzyn A, Osiadły M, Myslinska D, Wegrzyn G (2019) Autophagy-dependent mechanism of genistein-mediated elimination of behavioral and biochemical defects in the rat model of sporadic Alzheimer's disease. Neuropharmacology 148:332-346

Sassone J, Colciago C, Cislaghi G et al (2009) Huntington's disease: the current state of research with peripheral tissues. Exp Neurol 219: 385-397
Shannon KM, Fraint A (2015) Therapeutic advances in Huntington's disease. Mov Disord 30:1539-1546

Sharp AH, Loev SJ, Schilling G et al (1995) Widespread expression of Huntington's disease gene (IT15) protein product. Neuron 14:10651074

Tousley A, Iuliano M, Weisman E, Sapp E, Richardson H, Vodicka P, Alexander J, Aronin N, DiFiglia M, Kegel-Gleason KB (2019) Huntingtin associates with the actin cytoskeleton and $\alpha$-actinin isoforms to influence stimulus dependent morphology changes. PLoS One 14:e212337

Wu HC, Hu QL, Zhang SJ, Wang YM, Jin ZK, Lv LF, Zhang S, Liu ZL, Wu HL, Cheng OM (2018) Neuroprotective effects of genistein on SH-SY5Y cells overexpressing A53T mutant $\alpha$-synuclein. Neural Regen Res 13:1375-1383

Yang YP, Hu LF, Zheng HF, Mao CJ, Hu WD, Xiong KP, Wang F, Liu CF (2013) Application and interpretation of current autophagy inhibitors and activators. Acta Pharmacol Sin 34:625-635 